\title{
Lesion recognition and cleavage of damage-containing G-quadruplexes by DNA glycosylases
}

\author{
Aleksandra A. Kuznetsova \\ Institute of Chemical Biology and \\ Fundamental Medicine of the SB RAS \\ Novosibirsk, Russia \\ sandra-k@niboch.nsc.ru
}

\author{
Olga S. Fedorova \\ Institute of Chemical Biology and \\ Fundamental Medicine of the SB RAS \\ Novosibirsk, Russia \\ fedorova@niboch.nsc.ru
}

\author{
Nikita A. Kuznetsov \\ Institute of Chemical Biology and \\ Fundamental Medicine of the SB RAS \\ Novosibirsk, Russia \\ Nikita.Kuznetsov@niboch.nsc.ru
}

\begin{abstract}
Human telomeres contain repeated TTAGGG elements, in which the $3^{\prime}$ exposed strand may adopt a Gquadruplex (Q4) structure. In addition, more than $40 \%$ of human genes have been found to contain at least one sequence near the promoter regions, which potentially could form a quadruplex structure. The guanine-rich regions of telomeres are hotspots for oxidation forming 8-oxoguanine, thymine glycol as well as abasic sites (AP-sites), the lesions that are handled by the base excision repair (BER) pathway. The first step in BER is initiated by DNA glycosylases, which locate damaged DNA bases in the large amount of normal DNA. DNA glycosylases catalyze excision of the abnormal base and produce an AP-site. However, the features of DNA repair processes in non-canonical structures, including quadruplexes, are still poorly understood. Therefore, the purpose of this work was a comparative analysis of the efficiency of the removal of damaged nucleotides from Gquadruplexes by human BER enzymes. We analyzed activity and substrate specificity of 8-oxoguanine-DNA glycosylase (OGG1), endonuclease VIII-like 1 (NEIL1), endonuclease III (NTH1) to their specific damaged nucleotides. As the control prokaryotic formamidopyrimidine-DNA glycosylase (Fpg) and endonuclease VIII (Nei) were used. Direct detection of the product formation by PAGE allowed to estimate the efficiency of enzymatic conversion of damaged G-quadruplexes under the same experimental conditions. The stages of formation of enzyme-substrate complexes and catalysis were analyzed by stopped-flow technique, which allows to register conformational rearrangements of model DNA substrates.
\end{abstract}

Keywords - Base excision repair, DNA glycosylases, G-quadruplex

\section{Introduction}

Endogenous and exogenous agents such as highly reactive cell metabolites, external environmental compounds, UV or ionizing irradiation continually damage cellular DNA. The major sources of endogenous DNA damage are reactive oxygen species (ROS). ROS generate a variety of DNA base lesions, including 7,8-dihydro-8-oxoguanine (8-oxoguanine, oxoG), thymine glycol (Tg), 2,6-diamno-4-hydroxy-5formamidopyrimidine (Fapy-G), 4,6-diamno-5formamidopyrimidine (Fapy-A) and many others [1].

Most of this endogenous burden is handled by the base excision DNA repair (BER) pathway. BER involves several DNA $N$-glycosylases specific for removing a wide array of oxidized, alkylated or deaminated bases, and in some cases mispaired normal bases, which yields abasic (AP) sites [2].

Beyond the canonical duplex form, DNA can populate a wide range of states, from single stranded conformations to four-stranded arrangements. Guanine-rich nucleic acids can fold into the non-B DNA or RNA structures called Gquadruplexes (Q4). G-quadruplexes are four-stranded secondary structures formed by particular G-rich nucleic acid sequences. They result from the stacking of multiple stable
"G-quartets", planar arrangements of four guanines held together by Hoogsteen-type hydrogen bonding and further stabilized by monovalent cations (generally $\mathrm{K}^{+}$or $\mathrm{Na}^{+}$). The topologies of G-quadruplexes can be classified into several types: antiparallel, parallel, and hybrid. Usually, the syn/antiglycosidic conformation of guanines is considered to be an important factor in the G-quadruplex structure folding. Structure and function of G-quadruplexes have become an area of great interest. Recently, they have been associated with some human genetic neurodegenerative diseases [3]. Gquadruplexes are also believed to be important for telomere maintenance [4], DNA replication, genome rearrangements, DNA damage response, chromatin structure, RNA processing and transcriptional or translational regulation.

Despite the important roles that G-quadruplexes play in telomere biology and gene transcription, the features of DNA repair processes in non-canonical structures, including quadruplexes, are still poorly understood. Therefore, the purpose of this work was a comparative analysis of the efficiency of the removal of damaged nucleotides from Gquadruplexes by human BER enzymes. We analyzed activity and substrate specificity of 8-oxoguanine-DNA glycosylase (OGG1), endonuclease VIII-like 1 (NEIL1), endonuclease III (NTH1) to their specific damaged nucleotides. As the control prokaryotic formamidopyrimidine-DNA glycosylase (Fpg) and endonuclease VIII (Nei) were used. Direct detection of the product formation by PAGE allowed to estimate the efficiency of enzymatic conversion of damaged G-quadruplexes under the same experimental conditions. The stages of formation of enzyme-substrate complexes and catalysis were analyzed by stopped-flow technique, which allowed to register conformational rearrangements of model DNA substrates.

\section{Results and Discussion}

Several model DNA substrates were used. The model Gquadruplexes contained $\mathrm{Tg}$ residue at the loop region $(\mathrm{Q} 4-\mathrm{Tg})$ or oxoG residue at the middle of GGG (Q4-oxoG). Also control G-quadruplex without damage (Q4) and control duplexes with $\mathrm{Tg}$ or oxoG $(\mathrm{Tg} / \mathrm{A}$, oxoG/C) were used for comparison ofg cleavage data.

\section{Structural analysis of G4 sequences by circular dichroism (CD) spectroscopy}

Firstly, we analyzed the ODN structures by CD spectroscopy under several buffer conditions including those required for the enzymatic assays. The DNA sequences were folded in the quadruplex buffer containing $\mathrm{K}^{+}$, in which the ODN without a lesion had a hybrid quadruplex structure. The CD spectrum of the unmodified quadruplex Q4 has shown two pronounced maxima at $290 \mathrm{~nm}$ and $265 \mathrm{~nm}$. It should be noted, that the control G-quadruplex and quadruplexes containing $\mathrm{Tg}$ at the loop region gave similar CD spectra. The thermal melting of these substrates did not gave significantly 
reduced Tm values: $66^{\circ} \mathrm{C}$ for control Q4 and $58-62^{\circ} \mathrm{C}$ for modified Q4-Tg.

In contrast, the ODN with oxoG in the center G-quartet showed a dramatic change in the CD spectra compared to the native hybrid fold, namely, a maximum peak at $263 \mathrm{~nm}$ and a minimum at $245 \mathrm{~nm}$, that are characteristic of parallel Gquadruplex structures adopted by telomeric sequences in molecular crowding conditions. Nevertheless, the Tm values of quadruplexes containing oxoG were $70-75^{\circ} \mathrm{C}$. These data suggest the coexistence of parallel and other types of Gquadruplex conformations for Q4-Tg and Q4-oxoG, with a clear prevalence of the parallel conformation in the case of oxoG containing substrates.

\section{Glycosylase activity}

The model 17-nt duplexes containing a specific damage oxoG or $\mathrm{Tg}$ were used in order to compare the glycosylase activity towards G-quadruplex targets. Fpg, Nei and NEIL1 led to formation of the reaction product corresponding to the $\beta, \gamma$-elimination, while OGG1 and NTH1 led to formation of the reaction product corresponding to the $\beta$-elimination.

The interaction of Fpg with Q4-oxoG in $140 \mathrm{mM} \mathrm{KCl}$ led to the formation of several cleavage products, whereas in 50 $\mathrm{mM} \mathrm{KCl}$ the cleavage of DNA backbone occurred preferably at the oxoG residue. It should be noted that interaction of OGG1 with Q4-oxoG did not lead to the substrate cleavage at all. The interaction of Nei and NEIL1 Q4-Tg led to formation of the cleavage products at 140 and $50 \mathrm{mM} \mathrm{KCl}$, but the cleavage was more efficient in $50 \mathrm{mM} \mathrm{KCl}$. It should be noted that NTH1 cleaved Q4-Tg only at $50 \mathrm{mM} \mathrm{KCl}$.

Kinetic analysis of OGG1 and Fpg interaction with oxoGsubstrates

One of the most important goals in the study of DNA repair is the elucidation of the enzymatic mechanism that provides highly precise recognition of the damaged base and its removing from DNA. Time-dependent stopped-flow experiments were carried out using 17-nt duplex substrates containing a single lesion at the sixth position from the 5 -end and G-quadruplexes. DNA substrates were labeled by aPu or FAM/BHQ1. It should be noted that we used two type of 17 nt duplex substrates ${ }^{\mathrm{F}} \mathrm{OxoG} / \mathrm{C}^{\mathrm{B}}$ and ${ }^{\mathrm{F}} \mathrm{OxoG} /{ }^{\mathrm{B}} \mathrm{C}$. The first one contained FAM and BHQ1 residues on the opposite side of the duplex, whereas in the second one the FAM and BHQ1 residues were located at the one side of the duplex. These substrates allow to register not only the substrate cleavage but also the bending of DNA in the course of catalytic complex formation.

The interaction of Fpg with ${ }^{\mathrm{F}} \mathrm{OxoG} / \mathrm{C}^{\mathrm{B}}$ led to the strong arising of FAM fluorescence up to 100 second, whereas the interaction of $\mathrm{Fpg}$ with ${ }^{\mathrm{F}} \mathrm{OxoG} /{ }^{\mathrm{B}} \mathrm{C}$ resulted in $\mathrm{FAM}$ fluorescence decrease up to 5 second (duplex bending) and then to FAM fluorescence increase up to 100 second (substrate cleavage). The same FAM fluorescence changes were observed for interaction of OGG1 with ${ }^{\mathrm{F}} \mathrm{oxoG} / \mathrm{C}^{\mathrm{B}}$ and ${ }^{\mathrm{F}}$ oxoG/ $/{ }^{\mathrm{B}} \mathrm{C}$ substrates.

The interaction of Fpg with Q4-oxoG ${ }_{17}{ }^{\mathrm{F} / \mathrm{B}}$ led to slow increase of FAM fluorescence probably indicating the slow cleavage of G4 quadruplex. Also we examined the Gquadruplex with oxoG and aPu located from the 3'- or 5'-side of damaged nucleotide. The intensity of emission of $\mathrm{aPu}$ is influenced by its environment; in particular, it is strongly quenched when $\mathrm{aPu}$ is stacked in a DNA duplex. The interaction of Fpg with $\mathrm{aPu}$ containing quadruplexes led to slow increase of $\mathrm{aPu}$ fluorescence intensity at the same manner indicating a shift of $\mathrm{aPu}$ to a more hydrophilic environment, revealing the destabilization of the Gquadruplex upon formation of complex with enzyme.

The interaction of OGG1 with Q4-oxoG did not cause effect on fluorescence of FAM. Whereas the OGG1 interaction with Q4-oxoG quadruplex with aPu located from the 5'-side from damage led to the slight increase of $\mathrm{aPu}$ fluorescence probably indicating the binding process.

Kinetic analysis of Nei, NEILI and NTH1 interaction with Tgsubstrates

The interaction of Nei with ${ }^{\mathrm{F}} \mathrm{Tg} / \mathrm{A}^{\mathrm{B}}$ led to the strong arising of FAM fluorescence intensity up to 300 second, whereas the interaction of Nei with ${ }^{\mathrm{F}} \mathrm{Tg} /{ }^{\mathrm{B}} \mathrm{A}$ resulted in FAM fluorescence decrease up to 0.1 second (duplex bending) and then to the increase of FAM fluorescence up to 200 second (substrate cleavage). The same FAM fluorescence changes were observed for interaction of NEIL1 with ${ }^{\mathrm{F}} \mathrm{Tg} / \mathrm{A}^{\mathrm{B}}$ and ${ }^{\mathrm{F}} \mathrm{Tg} /{ }^{\mathrm{B}} \mathrm{A}$ substrates. The interaction of NTH1 with these substrates led to increase followed by decrease of FAM fluorescence in both cases.

The interaction of Nei and NEIL1 with Q4-Tg led to slow increase followed by fast decrease of FAM fluorescence probably indicating the slow cleavage of quadruplex. At the same time the interaction of NTH1 with Q4-Tg only led to decrease of FAM fluorescence up to 100 seconds, indicating the binding process. For all enzymes Nei, NEIL1 and NTH1 the interaction with aPu-labeled quadruplexes did not led to significant change of $\mathrm{aPu}$ fluorescence, suggesting that complex formation has no effect on aPu fluorescence intensity when aPu residue located in the loop region of quadruplex.

\section{ACKNOWLEDGMENT}

This work was supported partially by Russian Foundation of Basic Research grant No 19-04-00012 and Russian State funded budget project of ICBFM SB RAS \# AAAA-A17$117020210022-4$

\section{REFERENCES}

[1] M.D. Evans, M. Dizdaroglu, M.S. Cooke, "Oxidative DNA damage and disease: induction, repair and significance", Mutat. Res., 2004, vol. 567, pp.1-61.

[2] D. Svilar, E.M. Goellner, K.H. Almeida, R.W. Sobol, "Base excision repair and lesion dependent subpathways for repair of oxidative DNA damage", 2011, Antioxid. Redox Signal., vol. 14, pp. 24912507,https://doi.org/10.1089/ars.2010.3466.

[3] A.R. Haeusler, C.J. Donnelly, G. Periz, E.A. Simko, P.G. Shaw, M.S. Kim, N.J. Maragakis, J.C. Troncoso, A. Pandey, R. Sattler, J.D. Rothstein, J. Wang, "C9orf72 nucleotide repeat structures initiate molecular cascades of disease", 2014, Nature, vol. 507, pp. 195-200.

[4] Y. Xu, "Chemistry in human telomere biology: structure, function and targeting of telomere DNA/RNA", Chem. Soc. Rev., 2011, vol. 40, pp. 2719-2740. 